\title{
Wound Infection Caused by Chromobacterium violaceum: A Case Report from a Tertiary Care Hospital in Bangladesh
}

\author{
Rumana Alim1, Sofia Andalib Safiullah1, Shaila Munwar1, Ishad Mazhar', Sifat Uz Zaman², \\ Md. Sarwar Bari ${ }^{3}$
}

${ }^{1}$ Department of Microbiology, Medical College for Women and Hospital, Dhaka, Bangladesh ${ }^{2}$ Infection Prevention and Control Division, Medlife Healthcare Limited, Dhaka, Bangladesh ${ }^{3}$ Department of Printing \& Publications, Dhaka, Bangladesh

Email: *alimrumana@gmail.com

How to cite this paper: Alim, R., Safiullah, S.A., Munwar, S., Mazhar, I., Zaman, S.U. and Bari, M.S. (2022) Wound Infection Caused by Chromobacterium violaceum: A Case Report from a Tertiary Care Hospital in Bangladesh. Advances in Microbiology, $12,83-89$

https://doi.org/10.4236/aim.2022.122007

Received: January 17, 2022

Accepted: February 21, 2022

Published: February 24, 2022

Copyright $\odot 2022$ by author(s) and Scientific Research Publishing Inc. This work is licensed under the Creative Commons Attribution International License (CC BY 4.0).

http://creativecommons.org/licenses/by/4.0/

\begin{abstract}
Chromobacterium violaceum is a Gram negative, facultative anaerobe, generally present in water, soil in tropical and subtropical regions. This bacterium is an emerging environmental pathogen that causes life threatening infection in humans and animals. It can cause wound infection, visceral abscess, septicaemia, meningitis, diarrhoea, UTI. It is associated with significant mortality due to severe systemic infection. As the bacteria have high spreading tendency leading to sepsis, early identification and prompt treatment is necessary. Here we report a case of Chromobacterium violaceum wound infection in a 9 years old male from Dhaka, who was successfully treated with combination of cefixime and flucloxacillin antibiotics as per culture sensitivity report.
\end{abstract}

\section{Keywords}

Chromobacterium violaceum, Wound Infection, Antibiotics

\section{Introduction}

Chromobacterium violaceum is free living, soil and fresh water, Gram negative, facultative anaerobe, catalase, oxidase positive, motile bacillus, which grows readily in simple nutrient agar, MacConkeys agar, blood agar, CLED media. The first human case was reported from Malaysia in 1927. A total of 150 cases have been reported worldwide, mainly from tropics, including 8 cases from India [1]. In 1905, Wooley first reported its pathogenic characteristics when he observed septicaemia in water buffaloes [2]. Modes of transmission are by intake of con- 
taminated water, exposure of wound and traumatic lesions to contaminated soil and water, insertion of urinary catheter or use of different medical equipment in hospital environment [3].

It has been reported in Bangladesh in 2017 and 2018 [3] [4]. Serious and fatal infections have been reported from Argentina, Australia, Brazil, Cuba, Nigeria, USA, Taiwan (China), Singapore, and Vietnam. In most cases route of entry through broken skin, following contamination with soil or water [5]. This bacterium produces a natural antibiotic called violacein, which is useful for treatment of colon and other cancers [5]. Violacein also has antibacterial, antiviral property. Antibiotic aztreonam (monobactam) is a natural metabolic product of Chromobacterium violaceum [6]. Infection in humans is not common; and mostly overlooked by physicians as this bacterium is rare. It is diagnosed clinically by purplish lesion and dark purple colored colonies in culture [7]. We have reported here a case of Chromobacterium violaceum infection in a 9 years old male child who gave history of trauma of right toe while playing outside.

\section{Case Presentation}

In June 2021, a boy aged 9 years was admitted to Medical College for Women and Hospital (MCWH), Dhaka, with history of trauma of right middle to be 15 days back while he was playing as shown in Figure 1. He was initially admitted to paediatrics ward. Later he was referred to surgery department of MCWH. He was treated with injection ceftriaxone and inj.flucloxacillin, injection ketorolac, and injection esomeprazole and oral paracetamol. He was operated on 21.6.21 at 11 am under local anaesthesia. The name of operation was wound debridement. On examination, appearance of wound was gangrenous. Surgeons at first thought it was necrosed tissue due to dark color of the wound, but later found healthy tissue, so amputation was not required. Chromobacterium violaceum produces pigment called violacein which is responsible for dark purplish wound color. The boy was otherwise healthy. His chest X-ray was clear and tuberculin test was negative. Complete blood count showed rise in total count of WBC (Table 1). The wound was swollen, tender \& there was discharge of pus. Pus was sent to microbiology laboratory for culture and sensitivity. Initially culture showed no growth of bacteria. A repeat culture was again sent to same laboratory, where there was growth of Chromobacterium violaceum. In microbiology laboratory, the sample was cultured in blood-agar, MacConkeys agar media showing typical dark purple colored colony (Figure 2). Microbiological and Biochemical test was done [8] (Table 2). Antibiotic susceptibility test was done by Kirby-Bauer disc diffusion method on Mueller Hinton agar media and result was interpreted according to Clinical Laboratory Standards Institute (CLSI) guidelines for Enterobacteriaceae Gram negative bacteria [9]. Antibiotic sensitivity revealed resistance to ceftriaxone, ceftazidime and sensitivity to ciprofloxacin, linozolid, meropenem, gentamicin, aztreonam and intermediate sensitivity to pipercillin tazobactam (Figure 3, Table 3). His dressing was done regularly under aseptic procedure and discharged from hospital after total 8 days of hospital 
stay. Oral medication of cefixime and ciprofloxacin were given at the time of discharge. The patient was advised to come to hospital for change of dressing.

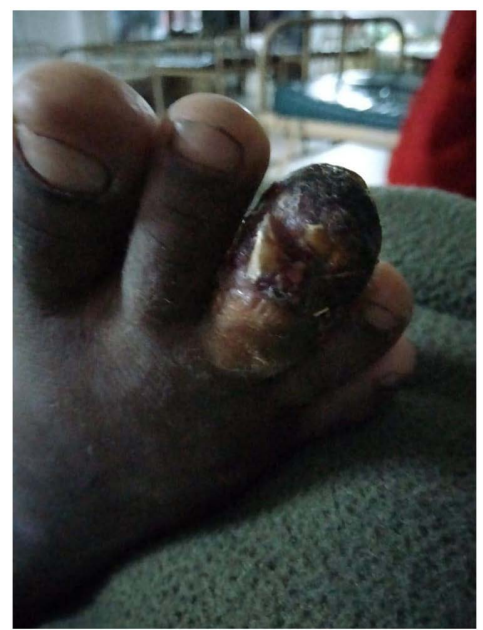

Figure 1. Injury of right middle toe showing dark purple colored pigment.

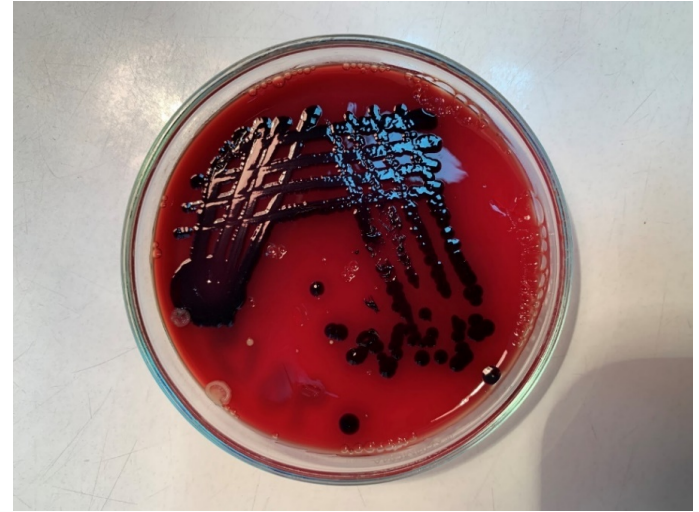

Figure 2. Growth of C. violaceum in blood agar media.

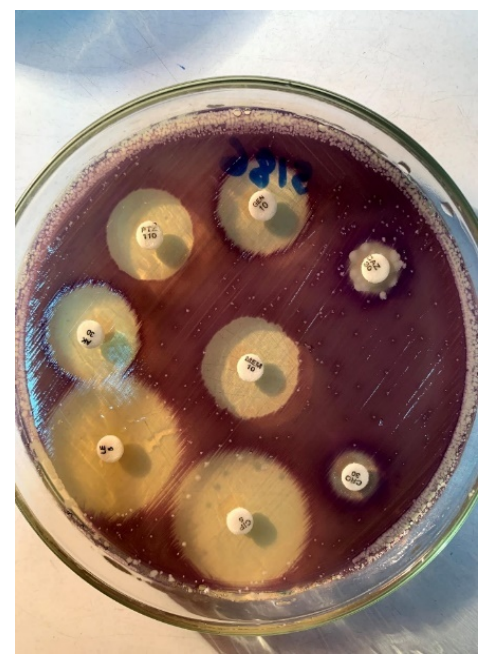

Figure 3. Antibiotic sensitivity test in Mueller Hinton Agar media: Kirby Bauer Disc Diffusion Method. 
Table 1. Hematology test result.

\begin{tabular}{cccc}
\hline Test parameters & Unit & Result & $\begin{array}{c}\text { Reference value } \\
{[14][15]}\end{array}$ \\
\hline Hb & gm/dl & 10.3 & Male: $10.7-14$ \\
ESR & mm/1 ${ }^{\text {st }}$ hr & 75 & Male: 0 - 10 \\
Total WBC count & cumm & 11,900 & $4500-13,000$ \\
N & $\%$ & 72 & $30-77$ \\
L & $\%$ & 20 & $14-50$ \\
M & $\%$ & 06 & $4-9$ \\
E & $\%$ & 02 & $0-5.8$ \\
B & $\%$ & 0 & $0-1$ \\
Total platelet & cumm & 336,000 & $1.5-4.5$ \\
RBC & M/ & 4.05 & $3.99-5.21$ \\
MCV & fl & 76 & $76-94$ \\
RDW & $\%$ & 5 & $11.6-14.8$
\end{tabular}

Table 2. Microbiological and Biochemical test reports.

\begin{tabular}{cc}
\hline Test & Interpretation \\
\hline Oxidase \& Catalase & Positive \\
MIU (Motility, Indole, Urease) & Motile, indole negative, urease negative \\
Simmons citrate & Negative \\
Gram stain & Gram negative rod \\
Culture (MacConkeys agar, blood agar) & Dark purple colored colony \\
Antibiotic sensitivity & Kirby Bauer technique, showing typical \\
(Mueller Hinton Agar Media) & dark purple colored colony.
\end{tabular}

Table 3. Antimicrobial susceptibility pattern.

\begin{tabular}{ccc}
\hline Antimicrobial agents & Interpretation & Zone of inhibition (mm) \\
\hline Ciprofloxacin & Sensitive & $\geq 21$ \\
Levofloxacin & Sensitive & $\geq 23$ \\
Meropenem & Sensitive & $\geq 15$ \\
Gentamicin & Sensitive & $\geq 17$ \\
Amikacin & Sensitive & $18-20$ \\
Pipercillin + tazobactam & Intermediate & $\leq 19$ \\
Ceftriaxone & Resistant & $\leq 17$ \\
Ceftazidime & Resistant & \\
\hline
\end{tabular}

\section{Discussion}

Chromobacterium violaceum is a rare bacterium present in environment that 
causes life threatening infections in humans and animals. In October 2017, a Bangladeshi farmer was hospitalized with high grade fever due to an agricultural injury related wound infection [4]. Another case was reported at a tertiary care hospital in Dhaka, Bangladesh, where $C$. violaceum was isolated in a 40 years female, who was diagnosed as pyrexia of unknown origin. The patient was later intubated and kept on mechanical ventilation as she developed pulmonary hemorrhage, hematuria and septic shock [3]. The patient ultimately died due to multiorgan failure. According to different review, $C$. violaceum cases were reported from 5 continents, showing the worldwide distribution. $C$. violaceum is distributed in tropics between $35^{\circ}$ latitude in the north and south [7]. From different review, $C$. violaceum infection cases predominantly occurred in young male patients, indicating group of people who are active outdoors and prone to injury [1]. Most common routes of infection are contact with water, through skin injury or ingestion [10]. It can be transmitted through inhalation of dust outdoors causing pulmonary infection with $C$. violaceum. This bacterium can cause nosocomial infection and be found in hospital equipments such as.catheters [11] and venturi masks [12]. C. violaceum has been reported from wound over scalp [5] and urinary tract infection and septicaemia in India [6]. C. violaceum infection was also reported from patients in Nepal causing upper UTI [11].

Our patient was 9-year boy, had injury while playing, so acquired infection most probably from soil. The patient was otherwise healthy. He responded well to combined antimicrobial treatment (combination of cefixime and ciprofloxacin) and surgical debridement. He was cured and discharged from hospital with follow-up. He was sensitive to Ciprofloxacin, Meropenem, Gentamycin, Amikacin. Intermediate sensitivity to Pipercillin + Tazobactam, resistant to $3^{\text {rd }}$ generation Cephalosporins-Ceftriaxone, Ceftazidime (Table 3). C. violaceum is usually resistant to many antimicrobials but sensitive to Imipenem, fluoroquinolones, gentamicin, tetracycline, and trimethoprim-sulfamethoxazole [13]. With rapid diagnosis and timely treatment patient was treated successfully. In previous case reports $C$. violaceum has led to fatal infection and death. Early detection with initiation of proper antimicrobial therapy is critical for management of this emerging and life threatening infection. From different studies it is observed that $C$. violaceum is distributed in environment, but isolation rate is not so significant. $C$. violaceum is an emerging environmental pathogen that causes fatal infection in both humans and animals. It was observed in a Bangladeshi farmer who presented high-grade fever following agricultural related wound infection. Bacteriological and gene investigation revealed $C$. violaceum from wound discharge. The patient recovered after combined antibiotic treatment with meropenem and ciprofloxacin [3]. Another case was observed in Hongkong, in a 40-year-old man with wound infection, who was treated with multiple antibiotics but died soon after admission to the hospital. Epidemiological survey revealed $C$. violaceum as probable cause of infection [16]. So physicians should be aware of this bacterium in their practice especially in wound infections. 


\section{Conclusion}

Human infection with Chromobacterium violaceum is rare. This is the first time the bacterium was isolated in our institution. Rapid diagnosis and the use of appropriate antibiotic for treatment is life-saving. As the bacterium is rarely isolated there is lack of awareness and knowledge among clinicians and microbiologists regarding this bacterium. Further intensive research work needs to be conducted in this regard.

\section{Acknowledgements}

The authors would like to thank the department of surgery of Medical College for Women and Hospital, Uttara, Dhaka, Bangladesh for providing history and data for making this case report.

\section{Conflicts of Interest}

The authors declare no conflicts of interest regarding the publication of this paper.

\section{References}

[1] Pant, N.D. and Sharma, M. (2015) Urinary Tract Infection Caused by Chromobacterium violaceum. International Journal of General Medicine, 8, 293-295. https://doi.org/10.2147/IJGM.S89886

[2] Wooley, P.G. (1905) Bacillus Violaceous Manila, a Pathogenic Organism. Bulletin of the Johns Hopkins Hospital, 16, 89-93.

[3] Sharmin, S., Jahan, A.A., Kamal, S.M.M. and Sarkar, P. (2019) Fatal Infection Caused by Chromobacterium violaceum: A Case Report from Tertiary Care Hospital in Bangladesh. Case Reports in Infectious Diseases, 2019, Article ID: 6219295. https://doi.org/10.1155/2019/6219295

[4] Majumder, R., Sadique, T., Sen, D., Majumder, P., Rahman, T., Chowdhury, A., et al. (2020) Agricultural Injury-Associated Chromobacterium violaceum Infection in a Bangladeshi Farmer. American Journal of Tropical Medicine and Hygiene, 103, 1039-1042. https://doi.org/10.4269/ajtmh.20-0312

[5] Kumar, M.R. (2012) Chromobacterium violaceum: A Rare Bacterium Isolated from a Wound over the Scalp. International Journal of Applied and Basic Medical Research, 2, 70-72. https://doi.org/10.4103/2229-516X.96814

[6] Kaniyarakkal, V., Orvankundil, S., Lalitha, S.K., Thazhethekandi, R. and Thottathil, J. (2016) Chromobacreium violaceum Septicaemia and Urinary Tract Infection: Case Reports from a Tertiary Care Hospital in South India. Case Reports in Infectious Diseases, 2016, Article ID: 6795743. https://doi.org/10.1155/2016/6795743

[7] Alisjahbana, B., Debora, J., Susandi, E. and Darmawan, G. (2021) Chromobacterium violaceum: A Review of an Unexpected Scourge. International Journal of General Medicine, 14, 3259-3270. https://doi.org/10.2147/IJGM.S272193

[8] Howard, A.J. and Ison, C.A. (1996) Haemophilus, Gardnerella and Other Bacilli. In: Collee, J.G., Fraser, A.G., Marmion, B.P. and Simmons, A., Eds., Mackie and McCartney Practical Medical Microbiology, 4th Edition, Churchill Livingstone, New York, 460.

[9] Clinical and Laboratory Standards Institute (2016) Performance Standards for An- 
timicrobial Susceptibility Testing. Vol. 36, 26th Edition, Clinical and Laboratory Standards Institute, Wayne.

[10] Byamukama, D., Farnleitner, A.H., Kansiime, F., Manafi, M., Burtscher, M. and Mach, R.L. (2005) Contrasting Occurrence of Chromobacterium violaceum in Tropical Drinking Water Springs of Uganda. Journal of Water and Health, 3, 229-238. https://doi.org/10.2166/wh.2005.028

[11] Pant, N.D., Acharya, S.P., Bhandari, R., Yadav, U.N., Saru, D.B. and Sharma, M. (2017) Bacteremia and Urinary Tract Infection Caused by Chromobacterium violaceum: Case Reports from a Tertiary Care Hospital in Kathmandu, Nepal. Journal of Medical Case Reports, 2017, Article ID: 7929671.

https://doi.org/10.1155/2017/7929671

[12] Hagiya, H., Murase, T., Suzuki, M., Shibayama, K., Kokumai, T., Watanabe, N., et al. (2014) Chromobacterium violaceum Nosocomial Pneumonia in Two Japanese Patients at an Intensive Care Unit. Journal of Infection and Chemotherapy, 20, 139-142. https://doi.org/10.1016/j.jiac.2013.10.001

[13] Graevenitz, A.V., Zbinden, R., Mutters, R. (2021) Actinobacillus, Capnocytophaga, Eikenella, Kingella, Pasteurella and Other Fastidious or Rarely Encountered Gram-Negative Rods. In: Murray, P.R., Baron, E.J., Jorgensen, J.H., Landry, M.L. and Pfalier, M.A., Eds., Manual of Clinical Microbiology, Volume 1, 9th Edition, ASM Press, Washington DC, 625.

[14] Van Leeuwen, A.M. and Bladh, M.L. (2016) Textbook of Laboratory and Diagnostic Testing: Practical Application at the Bedside. F.A. Davis, Philadelphia, 142-157. http://onesearch.nihlibrary.ors.nih.gov/permalink/01NIH INST/g83mj/alma991001 178659704686

[15] Das, K.V.K. (2017) Textbook of Medicine. 6th Edition, Jaypee, The Health Sciences Publisher, New Delhi, 1057.

http://www.amazon.com/Textbook-Medicine-Kv-Krishna-Das/dp/9386056100

[16] Sie-Keung, E.M., et al. (2006) A Fatal Case of Chromobacterium violaceumsepticaemia in Hong Kong. The Southeast Asian Journal of Tropical Medicine and Public Health, 37, 1179-1182. 